Social workers' reflections on ethics in relation to adoption in the UK:
Everywhere but nowhere?

\begin{abstract}
Empirical research with social workers exploring their understandings and use of codes or ethical theories in practice remains under-developed in the UK. This paper, based on the British Association of Social Work (BASW) commissioned Enquiry into the role of the social worker in adoption with a focus on ethics and human rights, provides an important contribution in this context. The Enquiry engaged with a range of stakeholders and explored their perspectives on the adoption process, but the primary focus of this paper is on how ethics were understood and discussed by social workers. One hundred and five social workers participated in the Enquiry through questionnaires, interviews and group discussions and a thematic analysis of their data revealed important findings. For example, the social workers made no explicit reference to codes of ethics or specific ethical theories. However, some of the themes that emerge from the analysis support discussions in what is now a substantial international literature on the importance of recognizing 'ethics work' by social workers (Banks, 2016). Weinberg's (2009) explorations of moral distress and ethical trespass are also important and underdeveloped concepts that resonate with themes from the Enquiry and could support more ethically enquiring cultures within organisations and more broadly.
\end{abstract}

Keywords

Adoption, ethics, social, workers moral, distress

\title{
Introduction
}

Adoption involves the permanent legal transfer of children from one family to another. In the UK it has become an established disposal for children who come into the care system as a result of child protection concerns and, in England, has been actively promoted by Government ministers particularly since 2010 . Studies show that many children have benefitted from adoption (see for example Neil et al., 2013; Selwyn et al., 2014). However, its use in contemporary contexts has not been without controversy. The impact on families living in poverty, in a context when 'austerity' policies are increasing hardship and reducing supportive services, has been highlighted by a range of authors (Featherstone et al., 2018; Hood et al., 2016; Bywaters et al., 2018). Moreover, 
the majority of adoptions are made without parental consent leading to criticism from a number of European countries when their citizens have been subject to adoption procedures in the UK (Fenton-Glynn, 2016).

Such concerns have become framed as ethical and human rights issues and prompted the British Association of Social Work (BASW) to commission an Enquiry to explore the role of the social worker in adoption across the four nations in the UK (see Featherstone, Gupta and Mills, 2018). While the Enquiry heard from a range of those impacted by, or working in the area of, adoption the focus in this article is on the perspectives of social workers. Moreover, while it explored ethics and human rights, the concern here, because of space constraints, is on how ethics were understood and discussed by social workers.

Empirical research with social workers exploring their understandings of ethics and, in particular, how much their perspectives and decision-making are informed by codes or ethical theories remains under-developed in the UK and this Enquiry provides an important contribution in this context. Some of the themes that emerge support discussions in what is now a substantial international literature on the importance of recognizing 'ethics work' by social workers (Banks, 2016). Weinberg's (2009) explorations of moral distress and ethical trespass are also important and underdeveloped concepts that resonate with themes from the Enquiry and could, we suggest, support more ethically enquiring cultures within organisations and more broadly.

First, we highlight some key themes from the literature in relation to ethics and social work and then explore the background to the Enquiry and the methodology used. We discuss key findings and their implications for organisations and social workers themselves.

\section{Ethically speaking}

There is a substantial literature on ethics and social work (see review by Lonne et al, 2016) although there is a need for more empirically grounded studies as indicated. A key development in the literature has been the questioning of the helpfulness, or otherwise, of developing codes of ethics that are then used by individual social workers when making decisions. This questioning has been prompted both by the evidence that social workers do not use such codes and by theoretical challenges (Rossiter et al, 2000; Congress and MacAuliffe, 2006; Weinberg, 2016). Over time, there has been a concern to stress the need to move from 'codes to contexts' (see, for example, Weinberg and Campbell, 2014). Thus there has been an evolution from a focus on duties, obligations, principles and rules to looking at particular contextual moral attitudes, dispositions and relationships (see for example Banks, 2016 and Weinberg, 2009; 2016). This has meant a focus on supporting social workers to ground ethical issues and decision making concretely. This reflects wider and very complex 
developments in philosophical and sociological thinking often captured, if with a critical slant, within writings on post-modernism and post-structuralism (see Rosssiter et al, 2000).

Broadly speaking these developments mark a critical re-think of hitherto dominant approaches of deontology and teleology. A deontological perspective focuses on absolutist notions of duty or obligation. This is captured in universalist approaches such as the Kantian categorical imperative: 'So act as to treat humanity, whether in your own person or that of any other, never slowly as a means but also always as an end' (quoted in Banks 1995, p. 28). The individual is worthy of respect simply because he or she is a person, regardless of what he or she has done, or whether he or she is useful to others. Deontology is the ethical theory most associated with professional codes of ethics, reinforcing the logic that professionals are duty-bound to follow their ethical code and that ethical practice without universal guiding principles is inconceivable (Weinberg and Campbell, 2014).

By contrast, teleology's focus is on ultimate ends. Consequentialism gives weight, as its name suggests, to the consequences of our moral decisions and actions- the rightness or otherwise of an act is judged in terms of the ensuing consequences. Utilitarianism is a form of consequentialism that is concerned with the value of ends for the greater good; it is committed to maximising happiness by doing the greatest good for the greatest number. Evans and Hardy (2017) argue that ideas derived from this perspective underpin the evidence-based practice movement, which emphasizes that 'what matters is what works' and ethically, the right course of action is that which empirical studies suggest is most likely to achieve a particular good outcome.

In recent decades a range of critical perspectives have emerged to challenge the dominance of these approaches. For example, virtue ethics insists that what is needed is something more dialogical:

The right answers must be negotiated in context, with attention to the particulars of this family in this situation, not an appeal to what are inevitably fictitious universals held in place by expert discourses... technical fixes in other words (Featherstone et al, 2014, p. 43).

What makes a practitioner's behavior ethical is the intention behind it and the practitioner's desire to act ethically. Unlike the emphasis on reason in Kantian approaches as the motive for acting virtuously, for virtue ethicists our innate tendencies propel us to act virtuously - to follow the virtues of honesty, kindness and care (Webb, 2006). Virtue ethics highlight the importance of considering the unique nature of each individual encountered in practice in order to help them discern what might be good for them.

A further critical development has been relational ethics. Drawing upon feminist thinking, an ethics of care emphasises the interdependence of human 
beings and the importance of relationships in ensuring human beings flourish physically and psychologically. Ethical decision-making cannot, or should not, be based upon assumptions of rational individualism but rather should recognise the importance of context and relational connectedness (Orme, 20012). Orme argues that: 'A dialogical approach to justice challenges the binaries not only of care/justice but also those of public/private, carer/cared for....... traditional ethics heard a single voice of disembodied moral principles, feminist ethics listens to and hears multiple voices because it defines morality and moral knowledge as plural and heterogeneous' (p. 809).

Banks (2016, pp. 35-36) draws from a range of approaches including virtue ethics and the ethics of care, as well as her own empirical research with social workers, to argue for the need to broaden the domain of professional ethics beyond the traditional focus on dilemmas and individual decision-making requiring rational judgment and beyond conformity to codes of ethics and rules of ethical conduct (ethics as external regulation). Situated ethics places dilemmas and decisions in a broader social, political and cultural context and sees responsibility in a wider, more relational sense, beyond the isolated individual decision-maker. She has coined the phrase 'ethics work' defining it as the work practitioners do to see the wider political (macro) context of their practice and take responsibility for being ethical and acting ethically.

Weinberg (2009) is a Canadian scholar from within a situated ethics approach who has carried out empirical research into social work decision-making and ethics. She has explored two concepts, moral distress and ethical trespass, that are of particular relevance to the concerns of this article (Weinberg, 2009; 2016). She notes that in the field of nursing, a theoretical concept called 'moral distress' has developed that identifies the psychological and emotional pain that arises when professionals feel blocked from doing what they consider is morally correct, due to institutional constraints. She argues that moral distress needs to be distinguished from the concept of ethical dilemma. Ethical dilemmas concern two or more courses of action that are in conflict (and will potentially have both positive and negative consequences), each of which can be defended as viable and appropriate. By contrast, moral distress arises if one action is preferred and seen as morally superior, but the person feels blocked from pursuing it by factors outside the self. Weinberg argues the notion of ethical dilemma can eviscerate the political responsibility of social workers to be agents of change in unjust or inadequate situations. By contrast, moral distress involves perceptions of moral accountability and the degree to which a worker views herself as individually responsible or as restricted by circumstances.

Weinberg notes that a limitation is that the notion of moral distress carries with it the assumption that there is an appropriate way of behaving. However, the field of social work is filled with situations in which no correct response can be assured or entirely right. Sometimes, regardless of what stance an individual practitioner takes, both good and harm will follow. Workers contribute to dominant discourses of what is taken to be 'normal' and this power carries with 
it the potential to injure because some ways of being will be supported and others invalidated.

Weinberg notes this is the idea of ethical trespass; the harmful effects that inevitably follow not from our intentions but from our participation in social systems and processes. For example, social workers, as people charged with responsibilities to determine standards, through the construction of those standards, judge some actions as beyond the boundaries of what is acceptable. Thus individuals who act in certain ways can be disciplined for violations. Furthermore, the reality that social workers usually have responsibilities to more than one service user at a time, such as in a family situation so that social workers' actions may harm one person in order to ensure another is protected.

Practitioners cannot ever fully know the full extent of the consequences of their actions and thus are involved in ethical trespass. Consequently, workers must confront the harm inherent in their positions and be self-reflexive and humble in their practice.

Overall, as we explore further below, we consider the concepts of ethics work, moral distress and ethical trespass, may be of value in understanding some of the issues that emerged from the Enquiry and taking these forward.

\section{The Enquiry}

BASW commissioned this Enquiry in 2016. Its remit encompassed the four countries of the United Kingdom, England, Scotland, Northern Ireland and Wales and it was concerned with adoptions that are undertaken by local authorities for children in care and, therefore, with the role of the social worker from initial child protection processes to post-adoption.

A key aim of the Enquiry was to gather evidence from as broad a range of interested parties as possible. This was a challenging endeavour involving reaching out to very diverse groups of people with differing levels of knowledge, educational backgrounds and abilities. Thus it was important to develop a working definition of ethics that was accessible but not too leading. Given the complexity of the subject and the volume of scholarship attached, this did not prove an easy task.

The following statement was part of the briefing materials disseminated to encourage participation in the Enquiry:

In its broadest sense ethics is concerned with looking at what is the right thing to do and what ought to be done. Ethics help us consider the benefits of actions or decisions for individuals, groups or society in general and the importance of the values and principles behind our decisions. So it moves us beyond questions such as 'does this policy work?' and it makes us consider questions such as 'is this policy right?' Thus this enquiry offers an important opportunity to explore what the role of social worker should be and what kinds of dilemmas adoption can highlight. 
We will reflect on the limitations of this definition in later sections.

The Enquiry employed a variety of methods the majority of which were well established research methods. A questionnaire was hosted on the BASW website to be filled in by individuals or organizations; telephone or face-to face interviews were used either to substitute for written submissions or supplement them; a focus group was held with adopted young people and one with children's guardians in England; and face-to-face interviews were held with members of the judiciary. A scoping review of the literature was also carried out.

Additionally, influenced by developments such as the Care Inquiry (2013), multi-stakeholder seminars were used. These involved a wide range of stakeholders such as birth parents, adopted people, adopters and social workers came together to discuss the issues over a day or half day period. In total seven seminars took place, two in England, Scotland and Northern Ireland and one in Wales.

Ethical approval for the Enquiry was obtained from the University of Huddersfield. This included obtaining written consent from those who completed questionnaires, interviews and focus groups.

In terms of the numbers and range of respondents, social worker were the largest group (105), followed by birth family members (56), adoptive parents (44), adopted people (32), academics (24), related professionals (24), and organizations (13). The majority of participants (165) were from England, 57 from Scotland, 43 from Northern Ireland and 29 from Wales.

This paper focuses on the responses of social workers, including practitioners in child protection and adoption teams, local authority managers, independent reviewing officers and Children's Guardians. Most of the respondents were self-selected, although a specific approach was made to CAFCASS in order to include the perspectives of Children's Guardians, and participants to the seminars were invited because of their known interest in adoption. A limitation of the self-selection aspect of the study is that respondents are likely to be social workers with a particular interest in and strong views about the subject area.

A large amount of data was collected: questionnaire responses, transcripts of recordings of interviews or groups, and contemporaneously taken notes from seminars. The phases in thematic analysis (see Barbour, 2016) were followed and included: reading and re-reading the data in order to really get to know it; generating codes; developing themes and sub-themes and reviewing the themes.

The analysis was initially conducted separately by two members of the research team and then discussed. A very high level of congruence was found between the two analyses. All the transcripts, the notes on coding and the generation of 
themes were then shared within the research team and discussed collectively.

\section{Talking, or not talking, about ethics}

There was a lack of reference by social workers to codes of ethics in practice as has been noted above. There was also no explicit reference made to, or discussion about, different schools of ethics or how ethical decisions were resolved in specific cases drawing upon particular theories. However, although unarticulated, many of the social workers' observations seemed to reflect a commitment to relational ethics, particularly to the ethics of care. Social work respondents expressed concern that practices should ensure that all involved in adoption were able to avail of social work relationships that gave them the time and emotional resources they needed. The importance of developing relationships with children was stressed in particular. The need for time to build relationships based on trust and respect was mentioned frequently, but seen as increasingly hard to achieve in a context of scarce resources and high caseloads.

There was also evidence of engagement with other ethical theories, although not named as such. For example, different views were expressed by some of the respondents about ethical issues in relation to matching and introductory processes, such as adoption activity days where prospective adopters can interact with children who are waiting to be placed, and 'bump-into' meetings where children are taken to the park by their foster carers, who 'bump into' some 'friends' of the foster carers who are the prospective adopters who have just been matched/linked with the children. From a consequentialist perspective, some found these useful in finding placements and felt there is a great deal of effort put in by social workers into making these work for children and be as ethical as possible. Others, however, questioned the lack of honesty with children, reflecting deontology and virtue ethics.

Consequentialism was also evident in discussions about timescales that balanced the perceived benefits of shorter or longer timescales before decisions are made about permanent placements, as explained by a social worker:

I feel that there is an ethical dilemma faced when removing a child from the care of the family, and placing them with adopters. There is a conflict of interest between the child's timescales (which appears to be getting shorter) and giving parents an opportunity and the support to care for their children.

In the process of making recommendations in court proceedings, social workers are required to analysis the pros and cons of various options, and there was evidence of some struggling with how to make judgements about 'good outcomes':

What I have wrestled with is the impact of adoption on a young child who has already begun to develop a sense of their identity within the birth family. How 
much do we really know about what a young child of 3, 4 or 5 is thinking or feeling? And how much have we followed up such children at later stages of life and explored the impact (positive or negative) of adoption? My dilemma at such points has been whether or not the system will create a greater disadvantage to this child in the long run.

Houston (2010) suggests that although different schools of ethics have been hard to reconcile they need to be in a world where social workers have to engage with legal injunctions, procedural rules, the future consequences of decision making, issues that have a bearing on professional integrity and situations of care. This is clearly highly pertinent to the role of the social worker in adoption where it is necessary to work within legal and policy frameworks but where many of these are subject to interpretation and contested value perspectives.

Overall, as we discuss below, the strongest messages related not to codes of ethics or theories of ethics, but to the impact of wider organizational, policy and political contexts.

\section{Ethics work}

The responses of social workers included evidence of 'ethics work' as defined by Banks (2016) and explored above. Thus, there were many examples of social workers evaluating what they were doing or not doing within an interrogation of the political and organizational contexts in which they were working. Examples were given that can be categorised under three inter-related themes: resources and the impact of austerity; wider policy contexts and organisational structures and systems.

\section{Resources and the impact of austerity}

Social workers highlighted the impact of austerity measures resulting in increasing hardship for families and severe reductions to family support services. Such reductions were considered to be contributing to an increase in costly care proceedings. Care experienced parents were highlighted as a group raising particular ethical issues. These concerned the obligations of 'corporate parents':

In one situation a young couple who had been parented by the local authority had a child removed. The obligations of the local authority as corporate parents towards the parents were not considered, only the child. If we are bringing up children in local authority care and they become parents what are our obligations?

Women who had experienced domestic abuse were also seen as being unfairly treated due to limited resources and a risk-averse culture: 
My other concern is the gendered nature of adoption, and children being removed due to the mother's failure to protect. Mothers are then blamed when they re-establish the relationship or enter another relationship which is abusive, or with a schedule one offender. However, the reason for the violence perpetrated by the father is often not explored or addressed.

A lack of resources, once children had come into care, was seen as impacting on the effectiveness of services and the ability of individuals to act in ways they felt were ethical, including placing siblings together. The importance of children having a right to be heard and being helped to understand what could be a very confusing process was identified; however, this required time and that was often lacking. There was a consensus that post-adoption support needed improving for everyone. Thus ethical issues were raised in relation to adopters being left with having to manage traumatised children without adequate help and work with birth families post adoption was considered to be given particularly low priority.

A clear message came through that lack of resources for marginalized families and within the organizations in which social workers work led many to feel compromised in their ability to work in ways they considered ethical. Some respondents, social workers and others, linked this to wider questions about what is a 'good' society. For example, concerns were raised about how families in poverty are disproportionately subject to State practices of scrutiny and removal, as well as the gendered implications in the impact upon vulnerable women:

However, I do feel that with sufficient time and resources, some adoptions could be prevented. I feel that wider factors, such as poverty and housing issues and the difficulties they create, are not acknowledged. I also feel that we have a responsibility to those children in the system, who may go on to have children of their own, that they are given the support and therapy that they need in a timely manner.

\section{Wider policy influences}

Whilst austerity policies have impacted on all four countries, discussion about the other wider policy influences varied across the countries. In England some policy makers and politicians have promoted adoption as the 'gold standard' in recent years in a way not replicated in other parts of the UK. A message that came through from many of the English respondents was that the government policy of promoting adoption has become the 'logic' or 'truth'. One senior social worker described the impact as follows:

'Often at the early help points of transfer stage a case is seen as an 'adoption' case because adoption is so high profile. Permanence has been hijacked to equal adoption child's 'right' to adoption. Belief is 'safe certainty' of adoption and adoption becomes a 'runaway train' and impossible for individual social workers to stop' 
In such contexts decision-making becomes apparently straightforward using uncritical formulations of both deontological (the right thing to do) and teleological (best outcomes) approaches. However, the responses of many of the participants suggested that this was at the expense of a situated ethical approach that reflected the complexities of individual children's and families' circumstances:

I can also think of cases where children were really settled in foster placements and there was pressure to go for adoption - which resulted in another move for them.

Some of the English social work respondents also identified the policy of the 26 week limit for care proceedings as being problematic. With the focus of proceedings being mainly on assessment, it limited the opportunities to support families:

Often I find that pre-birth assessments in such cases where there are elder children in the care system are only done to gather evidence in respect of future adoptions. This is required in the sense that we need to evidence our decisions, however more often than not this is process driven practice which is only done to ensure there is up to date evidence regarding the parents' lack of capacity.

Adoption social workers reported pressure to approve and match within specified timescales, with their work and that of the organisation evaluated on this basis, resulting on occasions feeling rushed to make decisions that were very consequential:

Due to 'scorecards' / performance data, and the way culture has evolved within adoption practice, it seems as though the message for professionals (i.e. familyfinding social workers, as well as those on adoption panels) is that you should placing as many children as possible, with little (or not enough) consideration to the circumstances.

However, whilst not advocating for imposed timescales, Scottish and Northern Irish respondents highlighted how various policies and public institutions, particularly the court systems, contributed to unnecessary and damaging drift and delay for the child, inhibiting their ability to practice in ways that they felt were ethically sound:

Scotland (unlike in England), it can take a long time for decisions to be made and this is not in the child's best interests.

\section{Organisational structures and systems}

It was recognised that the fragmented way services are currently organised can result in families, birth and adoptive, dealing with many different social 
workers. The level of specialism and the splitting between adult and children's services can mean different workers engaging with different parts of the family often with different service priorities. This may encourage silo thinking on the part of social workers as they work with one part of the process and can lose a sense of the whole picture. This can inhibit the development of relationships and the possibilities of gaining a holistic picture of the child. Indeed, it is entirely possible that the social worker who places the child for adoption has never met the birth family. Fragmentation is compounded by problems in retaining staff in many Children's Services Departments and the resultant churn in the workforce:

The difficulty in supporting adopted children is that the turnover of social workers is so high there is rarely anyone left in the council who knows the child within a couple of years of the adoption.

Splitting within the system was also identified, with social workers often split between seeing themselves as the social worker for the child rather than the family, and a further level of fragmentation can get layered on that with the social worker for the child in opposition to a social worker who sees herself as the social worker for the adopters.

\section{Discussion}

It is of interest to note that a very limited number of social workers across the UK chose to participate in the Enquiry. We are not sure why this may be the case. In discussions we had with non-participants, both during and since the Enquiry, we noted some possible contributory factors but it must be stressed that these need further exploration. For some social workers their primary concern when thinking about their role in adoption was about what disposal was best for children and children's outcomes in the light of the evidence available. Thus we observed that a concern with 'what works best for children' dominated the discursive space. While we see attention to evidence as profoundly important and, indeed, a vital element of acting ethically, it does seem of concern that discussion could be so prematurely closed off especially given the evidence is not settled and is unlikely to be.

For others who refused to participate, adoption was self-evidently a 'good thing' and, indeed, the motives of BASW in commissioning such as Enquiry were a source of concern. Why was the issue being addressed? Indeed, it was considered almost insulting and part of a continued narrative to 'do down' social work to be holding such an Enquiry.

In terms of the actions social workers felt able to take when they recognised issues as ethically problematic, we noted that they considered the Enquiry itself a resource for ethical action but highlighted the limited room for action there was more generally. There seemed to be a sense that organizational cultures were mired in compliance especially in England with little evidence from social 
workers that they were encouraged to discuss and debate the complexities of their work.

However, we observed even among those who engaged with the Enquiry evidence of powerlessness or fatalism. Post adoption contact in Northern Ireland was, for example, subject to much criticism from social workers. The majority of judicial recommendations were not legally binding and, yet, they were apparently accepted by social workers irrespective of whether they agreed or not. This may highlight also an underexplored area in the Enquiry; how social workers felt about their power position vis-a-vis other professions and how they did or did not own and use their own power.

In the rest of this discussion we reflect critically on whether the literature on ethics opens up possibilities for a reframing of some key issues within social work. We also reflect on the limitations and strengths of engaging social workers in 'ethics talk'.

\section{Reframing key issues?}

As indicated earlier, Weinberg (2009) draws attention to the notion of 'moral distress' and asks whether it can be used to understand a range of concerns in social work in the area of child protection internationally. Epstein and Hamric (2009) suggest that there are three potential consequences of moral distress: professionals may become morally numbed to ethically challenging situations; they may engage in different ways of conscientiously objecting to the trajectory of the situation, with varying degrees of productive results; or they burnout. Indeed, moral distress has been linked to burnout, reduced job satisfaction, and high turnover in nursing in a number of studies (Corley, 2002; Epstein and Delgado, 2010).

Retention issues are a very serious concern in social work particularly in the areas of child protection and children in care, impacting considerably on the services provided for children and families. For example, a report by the Children's Commissioner for England (2018) found that problems around recruiting and retaining children's social workers, were key factors in determining how likely children were to face instability and disruption.

However, retention issues are not commonly explored through a moral distress lens (Weinberg, 2009). The evidence from the Enquiry suggests the possible utility of such exploration. As we noted we found social workers who were very concerned that they were being blocked from doing what they considered to be morally correct and organizational contexts that were not conducive to the highlighting of their concerns.

Weinberg (2009) also uses the concept of moral distress to interrogate the consequences of the change from social work being a 'practical-moral' activity to one that is 'rational-technical'. This is exemplified by the fragmentation of 
the process and the emergence of discrete tasks and roles. Therefore, different parts of the 'problem' or different stages of a family's journey through care proceedings are dealt with in different parts of the system and by different workers. As we noted above, there were concerns expressed by social workers about this fragmentation and the implications for developing holistic understandings of children's identities. Workers described their concerns at systems where a sense of the child's history and identity was dispersed through a bewildering system.

Interrogating the responses of social workers and other participants through the lens of moral distress raises some crucial questions about other possible consequences that warrant exploration. Corley (2002) concluded that to deal with moral distress there are nurses who detach psychologically, perform tasks in a ritualistic manner or refer to those further up the hierarchy. For social workers does the need to desensitize oneself from the impact of 'austerity' measures lead to a greater propensity to subscribe to discourses that individualise risk regarding families involved in the child protection system? Could the concept of moral distress be useful, therefore, in broadening our understanding of the emotional impact of child protection work on workers and the consequences for the relationships they feel able to build with families?

We agree with Corley (2002) who argues that naming and identifying moral distress is a crucial starting point to explore the impacts on individuals and organizations, and ways of addressing these consequences. Although it has negative consequences, it may also have a positive impact. The concept requires recognition that a disjuncture between what one wishes to do professionally and what one enacts will have emotional and psychological consequences. Adopting the concept of moral distress requires acknowledgement of the divergence between preferred and actualized selves, and enables self-reflexivity and critical dialogue with others about the reasons for, and consequences of, practitioners falling short of their best intentions and values.

The notion of moral distress does have limitations however (see Weinberg, 2009). It assumes the dichotomy of human agency and structure and, in so doing, can allow workers to sustain a stance of victimisation by the system. This can be problematic especially given the issues noted above about a sense of fatalism and powerlessness. Moreover, practitioners are not separate from their environment. Social institutions are the creation of human beings. Thus, every time an individual enacts practice in a particular way, what constitutes social work is constructed at that moment.

It can also reinforce the assumption, as already noted, that there is one right way of acting. However, the idea of 'ethical trespass' captures the complexity and pain of the reality that social work is filled with situations in which no correct response can be assured or entirely right. We consider the notion of ethical trespass opens up possibilities in relation to thinking about 
organizational cultures. It is customary, for example, to call for reflective learning cultures in organizations. Such calls are often couched in terms of their contribution to learning from mistakes within the recognition that errors are inevitable. The notion of ethical trespass carries the potential for opening up new territory in relation to the issue of harm and the vital importance of constructing cultures that are attuned to exploring this as fully as possible.

\section{Engaging in 'ethics talk': limitations and strengths}

Banks (2016, p.46) notes: 'In one sense the whole of everyday life is about ethics (ethics is everywhere), in another sense none of it is (ethics is nowhere in particular)'. Being everywhere and nowhere can mean discussions can get very stuck and have a cul-de-sac quality particularly in contexts as we discovered where there appeared to be a lack of engagement with the complexity to be found in the literature on ethics. Moreover, framing ethics as we did in the briefing (as outlined above) in terms of whether a policy was right or not may have encouraged rather abstract discussions as happened in some seminars certainly. For example, is it ethical to have children waiting in the care system? Is it ethical to delay decision making?

The need for a contextualised approach is underscored we would suggest by the evidence from such discussions: is this policy right in this context? While practice examples were offered by social workers, it was notable that this was not a major feature probably reflecting social workers' anxieties about confidentiality. We would suggest, however, that it may also reflect that there is not a culture of discussing specific practice issues in terms of ethics.

It may also reflect gaps in social work education and research in relation to ethics. As we have noted, the scholarship is extensive and has exercised a very wide range of thinkers over the centuries. It can be challenging as we found to make concepts accessible and this makes it imperative that it is taught well and allocated sufficient time on the timetable. We are unclear about whether this is happening and, to what extent, students are being supported to apply ethics in specific political and organisational contexts. Moreover, the lack of empirical research with social workers may mean that when it is taught it is taught in too abstract a way and, therefore, may not engage students sufficiently. We would suggest all these issues merit further exploration.

What problems are there, however, with using a language of ethics? The messages from the Enquiry highlight the need for further exploration especially in relation to whether couching discussion of policies and practices in relation to ethics can obscure questions of power and politics. We noted that invoking the term was a practice engaged in people espousing very different perspectives that could end in stalemate and thus could be unsatisfactory in terms of promoting further dialogue.

\section{Conclusion}


Empirical research exploring with social workers what they understand by ethics and how much their perspectives and decision-making are informed by codes of ethics or ethical theories remains under-developed in the UK and this Enquiry provides an important contribution in this context. Some of the themes that emerge support discussions in what is now a substantial international literature on the importance of recognizing 'ethics work' by social workers (Banks, 2016). Furthermore, explorations of moral distress and ethical trespass are, we suggest, important and underdeveloped concepts that resonate with themes from the Enquiry and could support more ethically enquiring cultures within organisations and more broadly and have the potential to reframe existing discussions on retention and burn out.

\section{References}

Banks, S. (1995) Ethics and values in social work, London, BASW/Macmillan.

Banks, S. (2016) 'Everyday ethics in professional life: social work as ethics work', Ethics and Social Welfare, 10 (1), pp. 35-52.

Barbour, R. (2014) Introducing Qualitative Research, London, Sage.

Bywaters, P., Brady, G., Bunting, L., Daniel, B., Featherstone, B., Jones, C., Morris, K, Scourfield, J., Sparks, T., Webb, C. (2018) 'Inequalities in English child protection practice under austerity: a universal challenge? Child and Family Social Work, 23 (1), pp. 53-61.

Care Inquiry (2013) Making not Breaking: Building relationships for out most vulnerable children, available on-line at: http://www.frg.org.uk/images/Policy_Papers/care-inquiry-full-reportapril-2013.pdf

Children's Commissioner England (2018) Stability Index 2018, available on-line at: https://www.childrenscommissioner.gov.uk/publication/stability-index2018/

Congress, E. and McAuliffe, D. (2006). Social work ethics: Professional codes in Australia and the United States. International Social Work, 49(2), pp. 151-164.

Corley, M. C. (2002) ‘Nurse Moral Distress: A Proposed Theory and Research Agenda', Nursing Ethics 9 (6), pp. 636-650.

Epstein, E.G. and Delgado, S. (2010) ‘Understanding and Addressing Moral Distress, The Online Journal of Issues in Nursing, 15 (3), Manuscript 1.

Epstein, E. G. and Hamric, A. B. (2009) 'Moral distress, moral residue, and the crescendo effect', The Journal of Clinical Ethics, 20(4), pp. 330 - 342. 
Evans, T. and Hardy, M. (2017) 'The ethics of practical reasoning-exploring the terrain', European Journal of Social Work, 20(6), pp. 947-957.

Featherstone, B., White, S. and Morris, K. (2014) Re-imagining child protection: towards humane social work with families, Bristol, Policy Press.

Featherstone, B., Gupta, A., Morris, K. and White, S. (2018) Protecting Children: A Social Model Bristol, Policy Press.

Featherstone, B., Gupta, A. and Mills, S. (2018) The role of the social worker in adoption- ethics and human rights: An Enquiry available at https://www.basw.co.uk/system/files/resources/basw_55505-10_1.pdf

Fenton-Glynn, C. (2016) Adoption without consent: Update 2016, Study for the Petti Commission, European Parliament, available on-line at: http://www.europarl.europa.eu/supporting-analyses.

Hood, R. Goldacre, A., Grant, R. and Jones, R. (2016) 'Exploring demand and provision in English Child Protection Services', British Journal of Social Work, 46(4): 923-941.

Lonne, B., Harries, M., Featherstone, B and Gray, M (2016) Practising ethically in child protection, London: Routledge.

Neil, B., Beek, M. and Ward, E. (2013) Contact after adoption: a follow-up in late adolescence, Centre for Research on Children and Families, Norwich: University of East Anglia.

Rossiter, A., Prilleltensky, I. and Walsh-Bowers, R. (2000), 'A postmodern perspective on professional ethics', in Fawcett, B., Featherstone, B., Fook, J. and Rossiter, A. (eds.), Postmodern Feminist Perspectives: Practice and Research in Social Work, London, Routledge, pp. 83-103.

Selwyn, J., Wijedasa, D. N., and Meakings, S. J. (2014) Beyond the Adoption Order: challenges, interventions and disruptions, Department for Education, available on-line at:

http://researchinformation.bristol.ac.uk/files/34004613/Final_Report_04_Nov_2014.pdf

Weinberg, M. (2009) 'Moral Distress', Canadian Social Work Review, 26 (2), pp. 139-151.

Webb, S. (2006) Social Work in a Risk Society, Basingstoke: Palgrave.

Weinberg, M. (2016) Paradoxes in Social Work Practice, Oxford, Routledge. 
Weinberg, M. and Campbell, C. (2014) 'From Codes to Contextual Collaborations: Shifting the Thinking About Ethics $\square$ in Social Work', Journal of Progressive Human Services, 49, pp. 25-37. 